\title{
Application of Image Segmentation to Identify In-flight Particles in Thermal Spraying
}

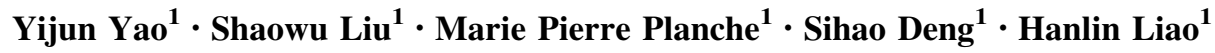

Submitted: 9 August 2021 / in revised form: 8 November 2021/Accepted: 8 November 2021/Published online: 1 February 2022 (c) ASM International 2022

\begin{abstract}
In thermal spray process, the characteristics of in-flight particles (velocity and temperature) play an important role regarding the microstructure of the deposit and thus the coating performances. The implementation of diagnostic devices is necessary to measure such characteristics. Many imaging systems and algorithms have been developed for identifying and tracking in-flight particles. However, these current image systems have significant limitations in terms of accuracy for example. One key to solving the tracking problem is to get an algorithm that can effectively distinguish different particles in the same image frame at the same time. This study aims to develop an algorithm capable of identifying a large number of in-flight particles sprayed by thermal process. The results show that the noise and vignettes could be successfully treated, particles are clearly recognized in the background, leading to properly measuring the sizes and positions of the particle versus time. The proposed algorithm has a higher recognition rate and recognition range than other algorithms, which will provide a reasonable basis for subsequent calculation and processing.
\end{abstract}

This article is an invited paper selected from presentations at the 2021 International Thermal Spray Conference, ITSC2021, that was held virtually May 25-28, 2021 due to travel restrictions related to the coronavirus (COVID-19) pandemic. It has been expanded from the original presentation.

Yijun Yao

yijun.yao@utbm.fr

1 ICB PMDM LERMPS UMR 6303, CNRS, Université de Bourgogne Franche-Comté, UTBM, Belfort, France
Keywords atmospheric plasma spray (APS) processing . image analysis processing · particle flight processing . particle flight analysing

\section{Introduction}

Thermal spraying is a surface engineering process that can deposit metallic or nonmetallic materials to elaborate functional coatings. Plasma spraying is one of the representative processes of thermal spraying, which generates an electric arc (plasma arc) by applying a voltage between the cathode and anode (nozzle).

When the plasma gas passes through the plasma arc, it is ionized by the arc and transferred to the plasma flame (Ref $1,2)$. At the same time, when the powder is transported to the vicinity of the nozzle through the powder supply pipe, the particles obtain energy from the plasma flame and are heated and accelerated. After a certain distance of flight, those high-energy particles hit on the substrate and deposit as a coating (Ref 3 ). Due to the concentrated energy beam and the high-temperature characteristics of the plasma flame, plasma spraying can melt many types of powders with high hardness and high melting point (Ref 4). The coating formation has the characteristics of low porosity, high-temperature resistance and high wear resistance. As a result, plasma spray can be used to manufacture coatings with various materials used in many industries.

The performance of the coating obtained by thermal spray largely depends on the operating parameters of the spraying, such as the choice of gas, the power of the arc and the properties of the powder. Among them, the characteristics of the in-flight particles and the coating performance show a strong correlation. Therefore, it is important to study the behavior of in-flight particles (Ref 5). Several 
studies have been made to address this issue. Many noninvasive measurement devices have been invented to observe the behavior of in-flight particles (Ref 6). For example, the research method concerning such behavior through the combination of numerical simulation and reverse spraying results is obtained by the observation system trends to be very popular ( Ref 7,8$)$. However, the results of such simulations invariably escape to a greater or lesser extent from reality. Therefore, it is necessary to expand the availability of information obtained through noninvasive observation systems to increase the possibility of conducting more research.

Currently, the information collected based on noninvasive observation methods for specific equipment is largely limited by the equipment used. For example, the DPV2000 diagnostic system (TECNAR, Canada) is widely used to obtain in-flight characteristics (velocity, surface temperature, diameter, trajectory, etc.) of each particle passing in the measurement volume (Ref 9). But using this system, the particle diameter could not be smaller than $10 \mu \mathrm{m}$ and the temperature should be higher than $1200 \mathrm{~K}$. Another limitation is that DPV2000 cannot obtain information near the nozzle exit, because the intense light emission of the plasma leads to saturate the photoelectric sensor (Ref 10). Moreover, DPV2000 cannot give out the vision image for further study.

Another type of noninvasive system based on the CCD camera can get a more intuitive image (Ref 11). However, as an optical imaging system, the camera will choose a large aperture, a high exposure speed and a high ISO value in order to capture the particles in-flight clearly, which will cause significant image noise (Ref 12 ). Due to the high light intensity of the flame jet and the broad spectrum of the plasma flame, the image in this area will inevitably be affected. Thus, it is difficult to directly identify in-flight particles in the obtained image with so many negative influences. Although there are many disadvantages in the CCD camera systems for observing the in-flight particles, with the development of image procession in the field of computer vision, many attempts have been performed by using this technology (Ref 13).

There are currently two main methods for extracting information of the in-flight particle present in the captured image. The first is to use the particle image velocimetry method to obtain the velocity of particle flow. The image correlation is used to determine the velocities of particles (Ref 14). However, since this method was originally used to observe fluids mixed with added reflective particles (Ref 15), the particle sizes are almost identical. Their positions also change over time with the fluid flow. Therefore, problems will arise when this method is applied to observe thermal spray particles. As the object of observation corresponds to a zone of sprayed particles, the size of in-flight particles is not identical in terms of granularity. During the spray process, particles gains accelerations due to the forces from the flow. These particles showing different velocities appear in the same area and interfere with the characteristics of the relevant zone. This algorithm will cause a lot of errors when performing the correlation detection of a specific zone, which greatly reduces the accuracy of the results. In addition, the velocity vector obtained by PIV consists of the velocity of the area but is not calculated from in-flight particles. Therefore, PIV is not suitable for studying the characteristics of in-flight particles of thermal spraying.

It might be ideal to accurately obtain the characteristics of each in-flight particle. However, the calculation load and the difficulty of obtaining this information are very large. Several studies attempted to get a trace of particles to make the process of tracking easier. The trace of particle is obtained by increasing the exposure time (Ref 16). As a result, the noise is effectively circumvented, and the traces of particles are beneficial for further research (Ref 17). However, as the particles imaging is blurred, the exact morphology of the observed particles is difficult to know. Moreover, the result is only valid if the traces of particles exhibit sharp edges, meaning therefore that the particles should be close to the focal plane of the camera. On the contrary, the particles far from the focal plane are almost unrecognizable due to their blurred edges.

So far, the precise identification of particles in flight is mainly made through manual identification. It also requires the researcher to greatly reduce the feeding rate and decrease the number of in-flight particles in the image to clearly distinguish each particle from each other. This method is inefficient, and it is necessary to use computer calculations as well as to develop unique algorithms for rapid particles identification.

The purpose of this study is to identify in-flight particles without prolonging the exposure time. Based on the algorithm development, it aims to get a stronger resistance to plasma and noise interference. First, the developed algorithm preprocesses the image to eliminate image defects and environmental interference. Then, it uses multi-level fuzzy edge detection to identify flying particles, which effectively avoids information loss and improves the accuracy of the algorithm.

\section{Methods and Experiments}

\section{SprayCam Device}

The noninvasive observation system used in this study is Control Vision Inc's SprayCam (Ref 18). The equipment is 
similar to a non-observation system consisting of a CCDbased camera, as shown in Fig. 1.

The control box in the SprayCam system is responsible for the energy supply of the entire system. The illumination box emits a laser beam with a wavelength of $905 \mathrm{~nm}$. Opposite to the illumination box is the sensor box: It receives the laser light from the illumination box and converts the signal into an electrical signal via the imaging element. Then, it sends the signal to the computer via the optical lens. After receiving the signal, the computer converts it into an image signal and stores it in the computer using the homemade software developed by Control Vision Inc.

As shown in Fig. 2, the XZ plane consists of the horizontal plane along which the centerlines of the two boxes for illumination and sensor are located. The XYZ coordinate system is set up, such as the nozzle location corre-

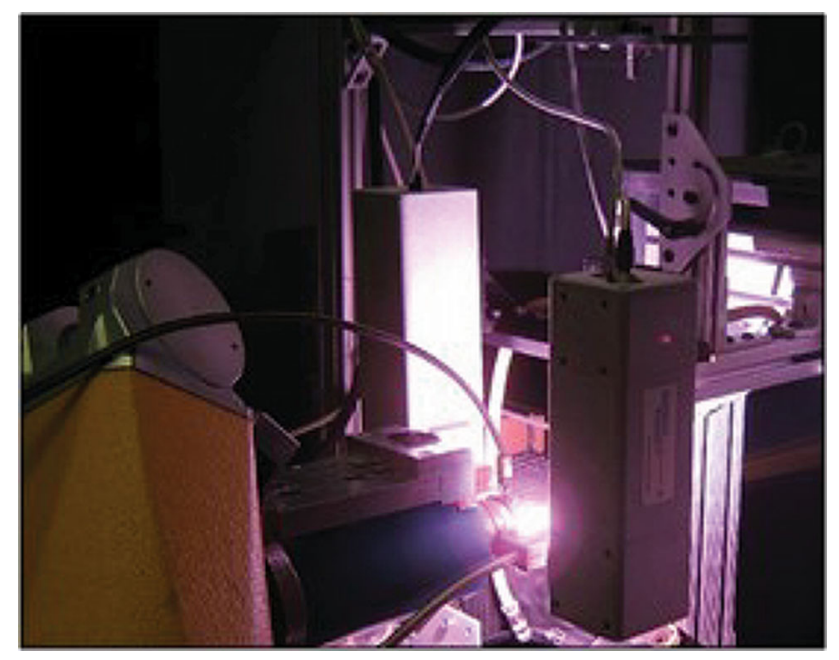

Fig. 1 Control Vision Inc's SprayCam system (Ref 19)

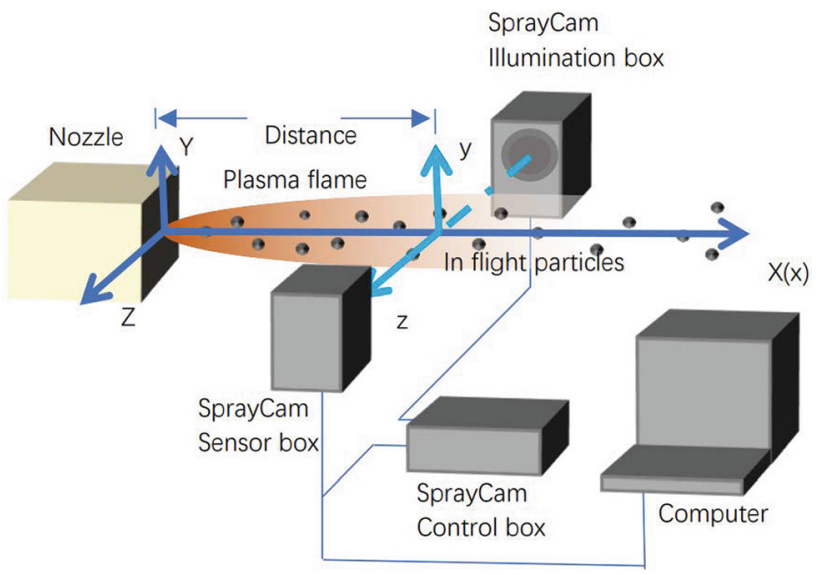

Fig. 2 Experimental diagram of the observation of in-flight particles in a plasma spray by using the system SprayCam sponds to the origin. The centerline of the two boxes corresponds to the focal plan. Then, the XYZ coordinate system is set up to get the intersection in between the centerlines of the two boxes and the spray centerline. That way, the distance between the origin of the two coordinate systems is the distance of the observation area from the nozzle.

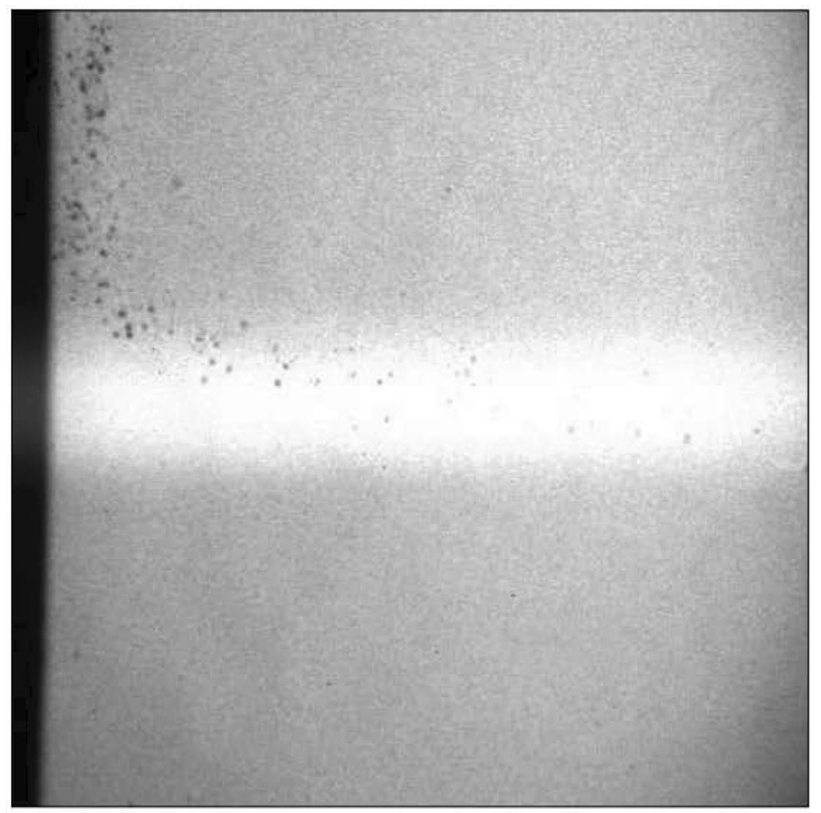

(a)

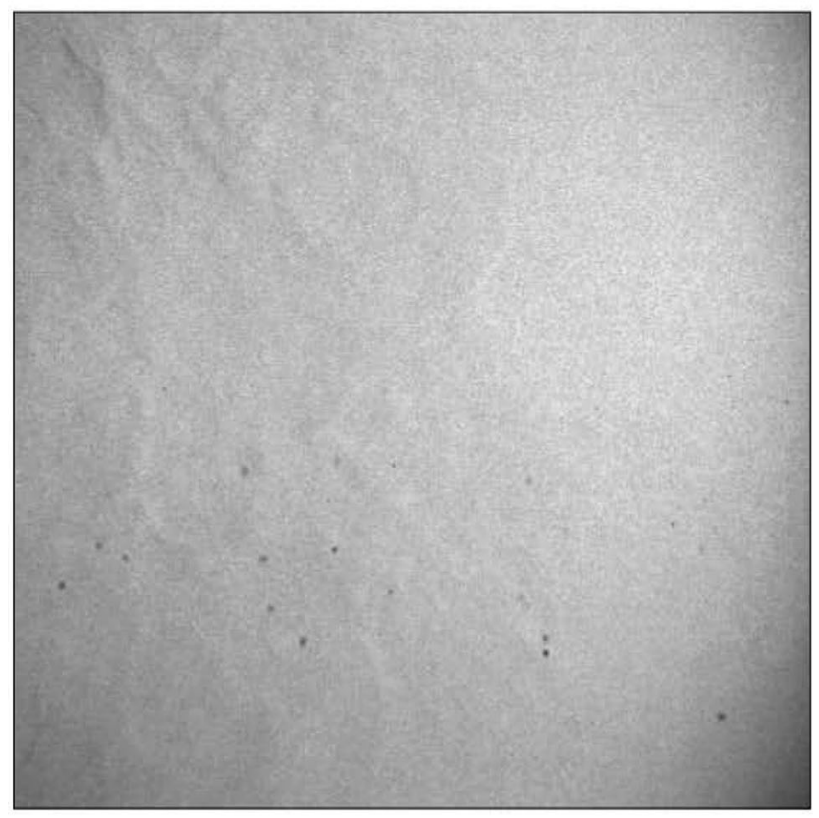

(b)

Fig. 3 SprayCam image of plasma spraying (a) $7 \mathrm{~mm}$ and (b) $47 \mathrm{~mm}$ downstream the nozzle 
Images of a typical plasma spray are displayed in Fig. 3. In Fig. 3(a), the black part on the left side concerns the nozzle. The whiter areas (plasma flow image) are due to defects in the SprayCam equipment. The sensor box accepts $905 \mathrm{~nm}$ wavelength laser as the imaging light. Due to the plasma beam having a broad spectrum and strong energy, the plasma jet contains high-intensity $905 \mathrm{~nm}$ light, which can interfere with the imaging process and cause the white areas. Fortunately, some particles are observed in the plasma jet. This means that the in-flight particles in the plasma flame can still be observed as long as an algorithm works to remove the plasma light interference. Besides, the interference of the flame with the image is significantly reduced on the right side of the image. In Fig. 3(b), the plasma interference is no longer visible. This distance does not act as a disturbed area for the DPV2000 and conventional CCD camera, demonstrating by the way the power of the SprayCam system based on a specific wavelength. Besides, for all these images, the vignette is severe. But the image processing techniques can be used to remove the vignette (Ref 20$)$.

Due to the distribution of the size, the in-flight particles captured do not have identical velocity. Also, as the inflight particles detected are not precisely distributed along the XY plane, they have different imaging patterns. Particles with clear edges and particles with blurred edges can be easily found in the image. This is due to the fact that the particle is not located in the focal plane.

According to existing algorithms, when a particle is far from the focal plane and has no visible edge, the algorithm could not recognize the object. So, in a first approach, only the objects close to the focal plane would be involved in the rest of the study, while objects far from the focal plane would be discarded. In that case, the applicability of such results is therefore limited.

Based on this discussion, the algorithm should:

- be able to remove the effects of lens vignette.

- be unaffected by the plasma jet.

- be capable to recognize in-flight particles in intense noise.

- capture particles far away from the focal plane as much as possible.

\section{Fixed Particles Image}

As the interference and defect in the spray image greatly disturbed the image, it is difficult to identify particles if the interfering factors are not well studied and eliminated. In addition, in order to better understand the imaging rules of the equipment and find out the method to propose interference factors, a group of effective data is needed. That means: A group of images with accurate particles position

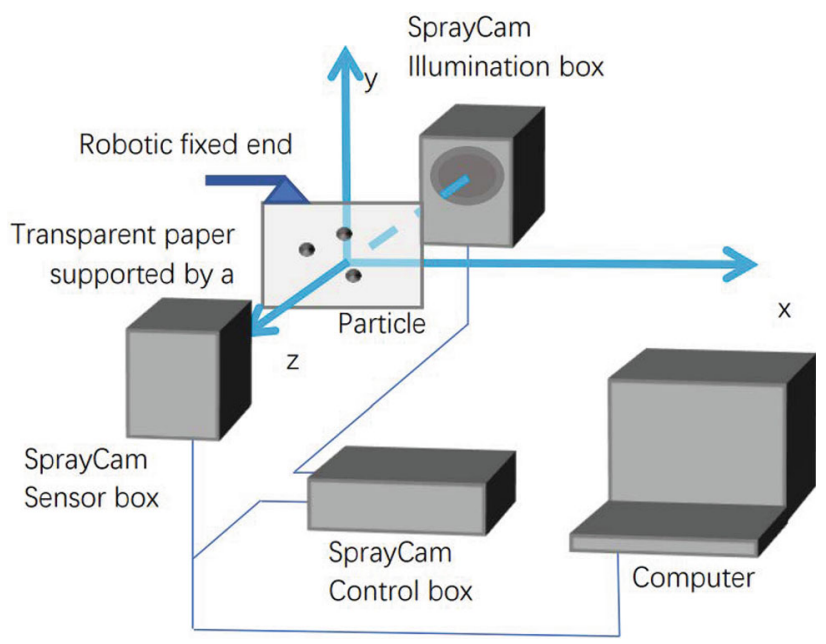

Fig. 4 Experimental setup using electrostatically fixed powders

is a good deal for this work. When particles are no longer uncontrollable factors, interference factors will be better identified and be eliminated. However, it is not easy to precisely control the position of the particles in the spray. To solve this problem, an experiment consists in simulating particles situation in-flight by fixing some particles on transparent paper.

In Fig. 4, the plasma spray equipment was replaced with a thin transparent paper supported by a rigid frame fixed itself onto the robot arm. Thus, the particles could be observed without any perturbation. When the robot arm moved so that the structure entered the measurement range of the SprayCam, the particles fixed on the transparent paper were imaged by the SprayCam. In addition, the transparent paper is divided into multiple areas by a square of 5 millimeters. These squares can make the experiment more effective.

As a powder commonly used for plasma spraying, $\mathrm{ZrO}_{2}$ was chosen as the powder for observation, with its $d(0.5)=$ $54.273 \mu \mathrm{m}$. Figure 5(a) shows the imaging results of the fixed particle experiment. The imaging result of the powder is represented by a series of pixels with grayscale values. And due to the low resolution of the equipment is not very high, the morphology of the powder is difficult to be accurately represented. Therefore, if other powders are chosen, the imaging results do not differ much. It can be assumed that the observation system based on this device cannot distinguish the difference between different powders. By observing the square's deformation, whether the transparent paper is parallel to the XY plane can be determined. The square area can also divide the particles into multiple observation areas, making it easier and faster to locate particles when developing algorithms.

The frame was moved by the manipulator in the z-direction, recording the position where the particles were 
Fig. 5 (a) Schematic of the imaging results. (b-e) Imaging results of the same area at different distances from the focal plane. (e) Imaging results of the same particles at different distances from the focal plane

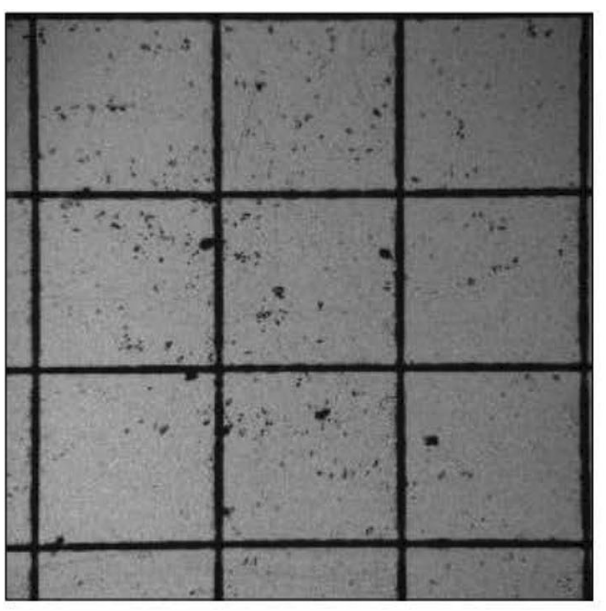

(a)

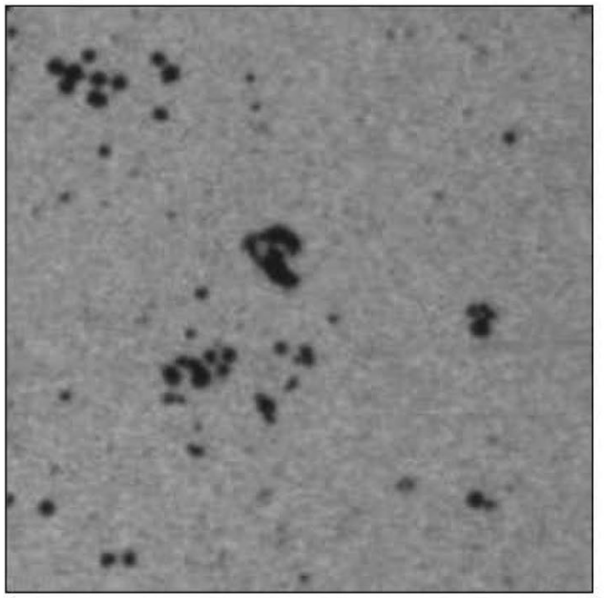

(c)

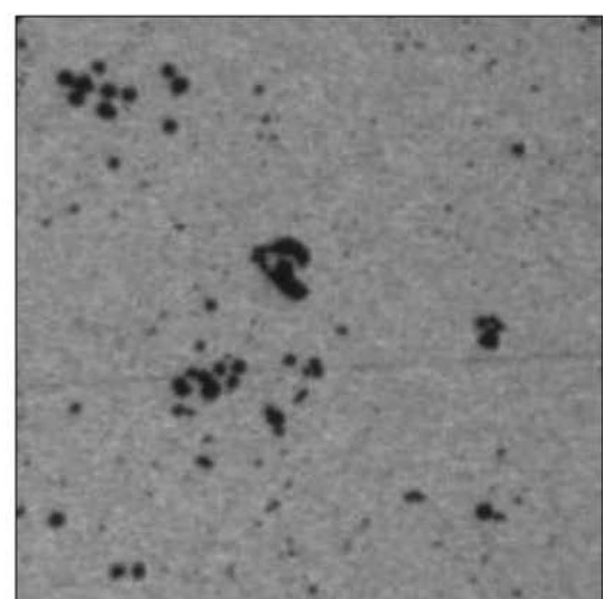

(b)

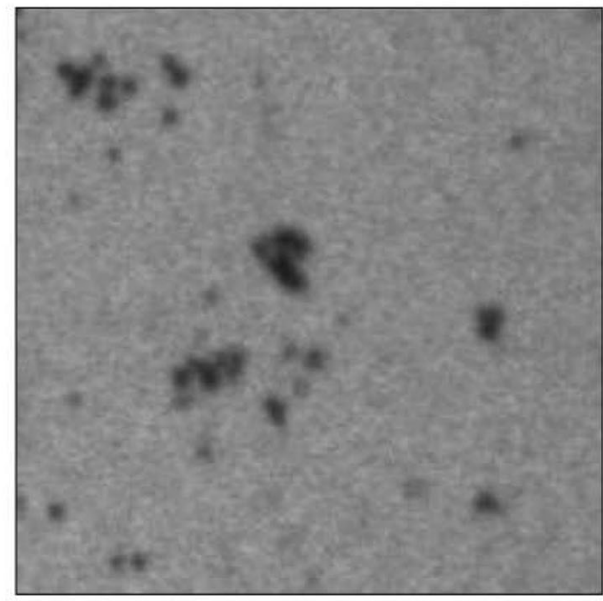

(d)

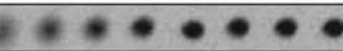

(e)

clearly imaged. Figure 5(b-d) shows the results of imaging particles in the same area at different distances. Figure 5(b) shows the image obtained when the particles on the transparent paper are on the focal plane. The result of their imaging has sharp edges. The impact of noise is serious. The robot moves a certain distance along the z-axis to obtain Fig. 5(c) and (d). Compared to the image in Fig. 5(b), the edges of the imaging result are blurred. In addition, the imaging of smaller particles starts to blend into the background and even disappear, making it more difficult to identify the particles. At the same time, the impact of noise is still very damageable. Figure 5(e) shows the result of imaging the same particle at different distances from the focal plane. The most central image is the particle image on the focal plane. The left and right sides correspond to a moving of the robot in the -z-direction, in one or the reverse direction (symmetrical from the focal plan). Therefore, a rule of SprayCam imaging can be summarized as follows based on the same particles. When the objects are close to the focal plane, their edges will be sharp. When they leave the focal plane, their edges begin to be blurred and gradually blend into the background. The imaging result of the object is also symmetric with respect to the focal plane.

Moreover, as observed, powder and dust were both adsorbed on the transparent. So, in this situation, a new requirement was established in the algorithm development in order to include dust identification: Dust would be regarded as an object that needs to be distinguished from the noise, resembling to the powder detected. 
Table 1 Parameters for capturing image

\begin{tabular}{ll}
\hline Plasma spray parameters & \\
Current intensity & $660 \mathrm{~A}$ \\
Plasma gas & $31 \mathrm{~L} / \mathrm{min} \mathrm{Ar}, 10 \mathrm{~L} / \mathrm{min}_{2}$ \\
Carrier gas & $2.6 \mathrm{~L} / \mathrm{min} \mathrm{Ar}$ \\
Powder feeder & $10 \%$ \\
SprayCam parameters & \\
Exposure & $10 \mu \mathrm{s}$ \\
Manual gain value & 548
\end{tabular}

\section{Plasma Spray Image}

In order to verify the efficiency of the algorithm, a spray image based on thermal spray is needed. Since this spraying method has more interference factors, it can better detect the inclusiveness of the algorithm. The parameters of spraying and SprayCam are synthetized in Table 1.

\section{Particles Identification}

According to the analysis of the SprayCam images, two main categories can be taken into account by the algorithm. One concerns the imaging defect due to the equipment, such as vignette and plasma interference, and the other provides from the interference of high-intensity noise for the identification of the particles. The algorithm also has a good recognition rate when dealing with particles close to the focal plane.

\section{Image Preprocessing}

The process of resolving the vignette and plasma jet impacts is defined as the image preprocessing process. The image preprocessing should effectively remove the imperfection of the image so that to identify the objects in the image. This process should be capable of separating the in-flight particles from the background even in the spray jet.

Regarding the images captured during the spraying experiment, the nozzle and the light conditions are relatively fixed, which can be considered that the stacking of a large number of images can get a static background without moving objects. The obtained background can help the algorithm to understand the vignette in the current area and let the computer know how to remove it.

The SprayCam images are grayscale images in such a way that each pixel corresponds to a value between 0 and 255. 0 represents Black and 255 represents White, respectively. For the pixel $(x, y)$ in the specified image, $f(x, y)$ is defined as its grayscale value, $N$ as the total number of pictures used for the background calculation. Figure $6(\mathrm{a}-\mathrm{c})$ shows three sprayed images taken at the same location. The background of $\mathrm{n}$ images is the average of the gray values of all images. For one pixel in the background $F_{\mathrm{bg}}(x, y)$ follows the following formula, $\mathrm{Eq} 1$.

$F_{\text {bg }}(x, y)=\frac{1}{N} \times \sum_{i=1}^{N} \sum_{j=1}^{N} f(i, j)$

The no-object background obtained by calculating $E q 1$ are shown in Fig. 6 (d).

After obtaining the background and subtracting it, this image should be inverted to remain only the objects and noise. The formula for the calculation is according to $\mathrm{Eq} 2$ :

$F(x, y)=255+f(x, y)-F_{\text {bg }}(x, y)$

The preprocessed images are shown in Fig. 7. Figure 7 (a) shows the result of the image near the nozzle. Figure 7(b) shows the result of preprocessing the spray image far away from the nozzle. Figure 7(c) shows the result of fixing the particle image. Figure $7(\mathrm{~d})$ shows the enlarged area shown in Fig. 7(c), which is the same as Fig. 5(b). It can be concluded that the vignette has been removed; meanwhile, the details of the objects have been perfectly preserved. In general, the preprocessing algorithm has greatly reduced most factors' interference and prepared relatively pure images for the particle detection process.

\section{Particle detection}

The preprocessed image has almost removed the vignette interference, which means that the range of effective detection has been enlarged to the full image instead of the middle of the image. Therefore, all the objects captured in the image are regarded as target objects that need to be detected.

As mentioned previously in Fig. 5(e), the edges of the objects change noticeably when the object is far away from the focal plan (Ref 21). Dependent on the position from the focal plan, the closer objects have sharper edge compared to farer objects presenting larger grayscale gradient on the edge. Thus, the standard algorithm that uses edge gradient to identify the objects does not work for the objects out of the focal plan. For example, it cannot distinguish objects from noise when a low gradient threshold is applied, or it cannot either recognize objects far from the focal plane when the gradient threshold is too high. Consequently, the edge detection algorithm based on the gradient threshold could not be suitable for this study. However, an algorithm based on the fuzzy edge detection algorithm can be used to detect the particles in the thermal spray process (Ref 22). 
Fig. 6 (a-c) Three spray particle images taken at the same position. (d) Stacked background without objects

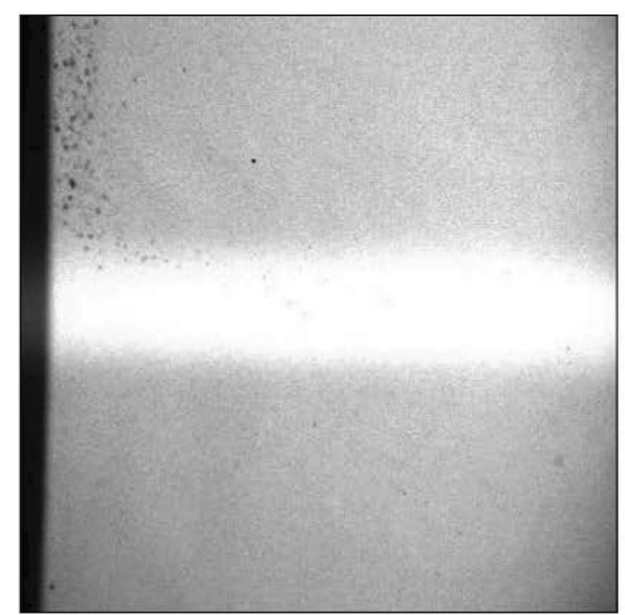

(a)

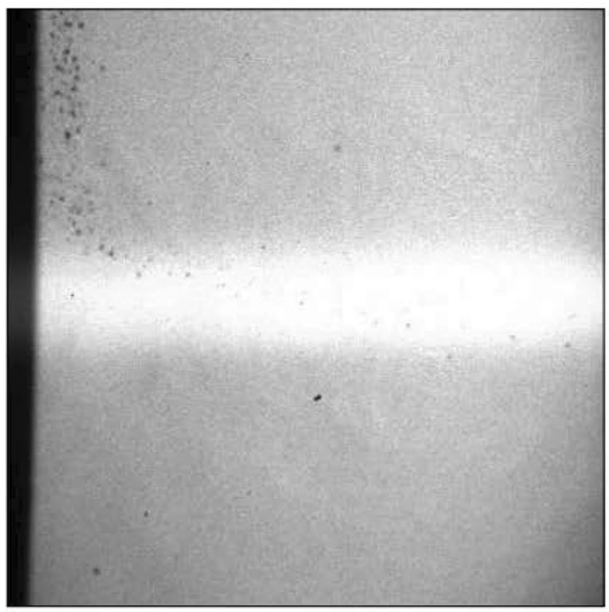

(c)

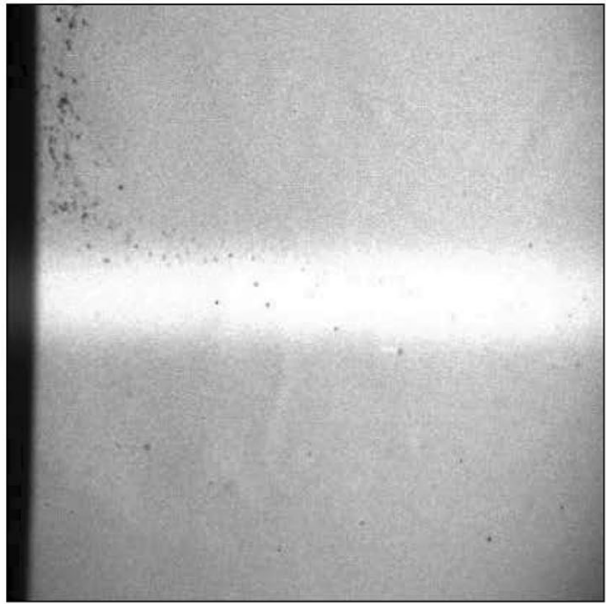

(b)

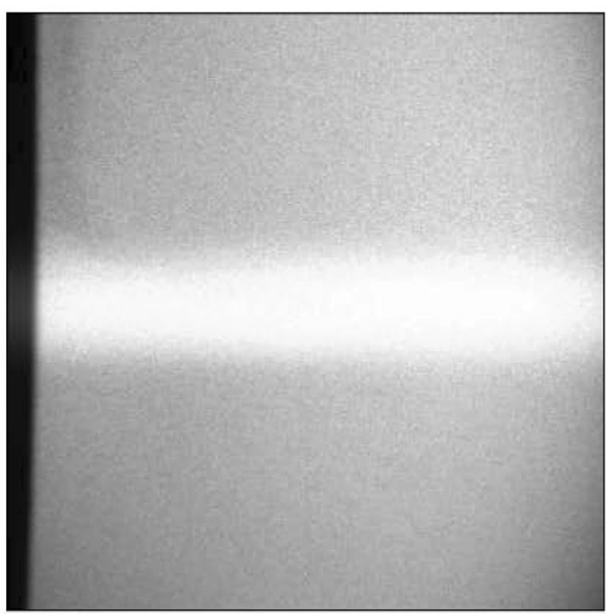

(d)
According to the computer image principle: Pixels considered as noise or background correspond to 255, representing the gray value of WHITE; pixels considered as object correspond to 0 , representing the gray value of BLACK.

\section{Algorithm procedure}

The algorithms for detecting objects consist of three main steps: multi-level fuzzy operation, object detection and merging results. First, the fuzzy operation is applied to the entire image pixel by pixel. That means the algorithm will calculate the average of the grayscale of all the pixels contained in a square (green window in Fig. 8) centered around the pixel studied $(x, y)$ according to a specific length ( $L=5$ in Figure 8). Then, this average gray value is set to the pixel studied $(x, y)$. This operation will be performed on all the pixels in the image to get a blurred image.

This calculation can be expressed by the following formula:
$G(x, \mathrm{y})=\frac{1}{\mathrm{~L}^{2}} \times \sum_{i=x-\frac{L-1}{2}}^{x+\frac{L+1}{2}} \sum_{j=y-\frac{L-1}{2}}^{y+\frac{L+1}{2}} F(i, j)$

where $F(x, y)$ is the gray value of the preprocessed image, $G(x, y)$ is the gray value of the blurred image, which can be considered as the average gray value of the rectangle of length. As an example, Fig. 7(d) is used as the input image, and the result obtained through Eq 3 is shown in Fig. 9.

In the second step, the obtained blurred gray value of this pixel will be compared to the gray level value of the pixel studied.

$g(i, j)=\frac{G(i, j)-F(i, j)}{\mathrm{G}(i, j)}$

If the result $g(x, y)$ is greater than the predefined value $\mathrm{K}$, the algorithm will identify the pixel $(x, y)$ as a pixel coming from an object and mark it as BLACK (0). Otherwise, it will be considered as a noise and will be marked as WHITE (255). The original image of Figure 5 
Fig. 7 (a-c) Image after preprocessing. (d) Image after preprocessing of the same area of Fig. 5(b)

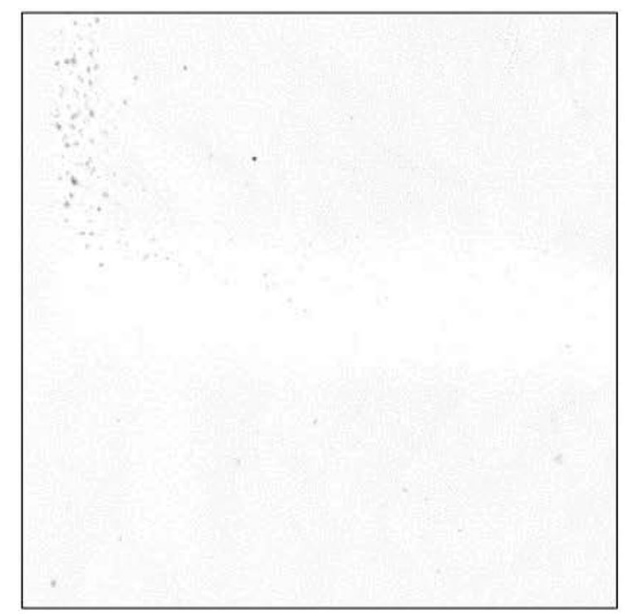

(a)

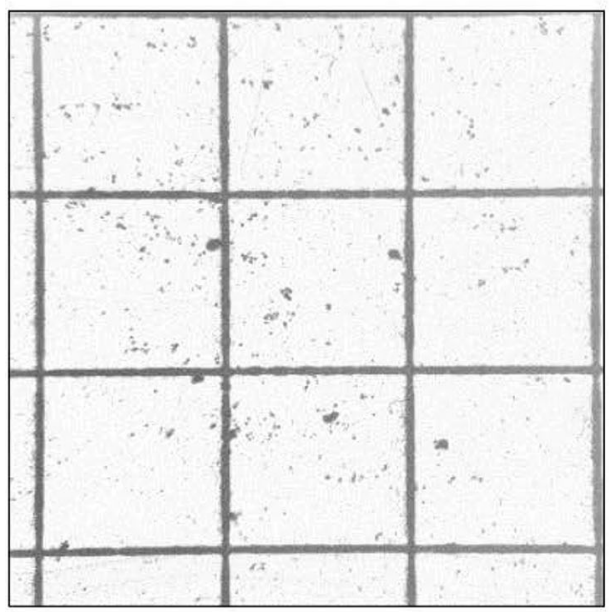

(c)

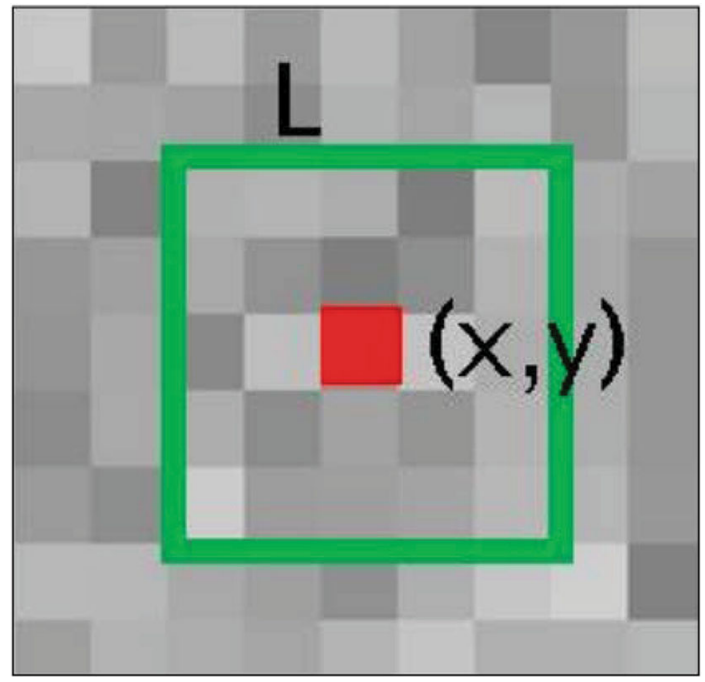

Fig. 8 Diagram of fuzzy operation with window sizes $L$ on the pixel $(x, y)$

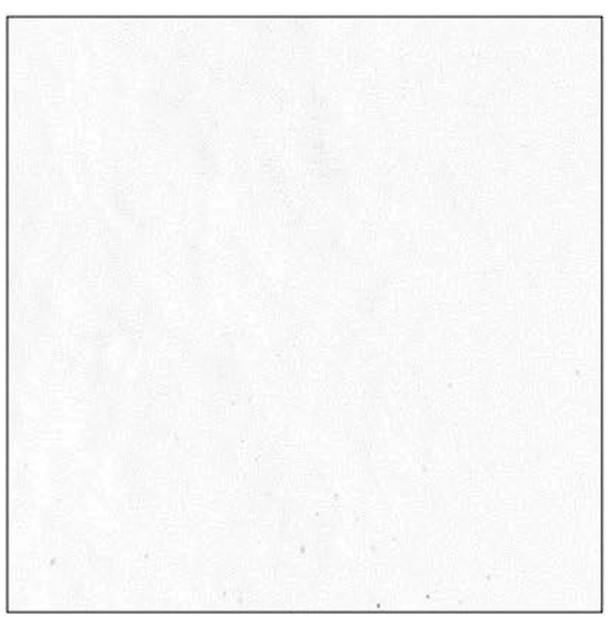

(b)

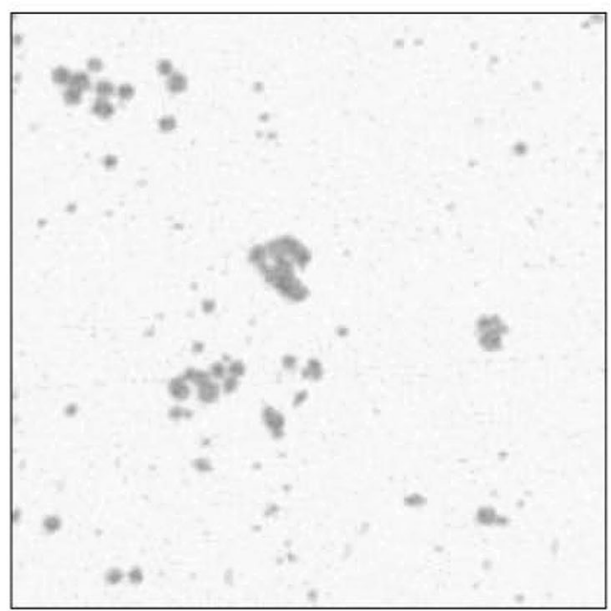

(d)

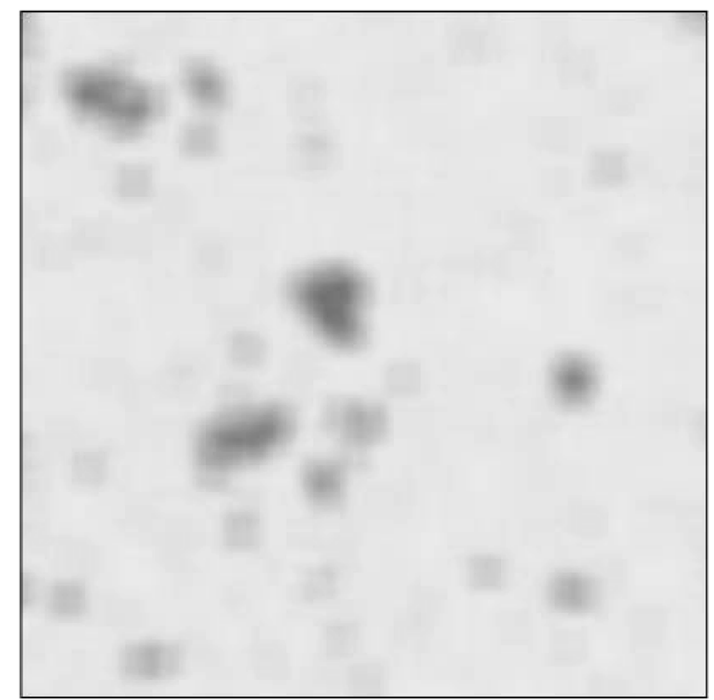

Fig. 9 Blurred result of Fig. 7(d) when $L=5$ 
Fig. 10 Detection results obtained when $K=0.2$, (a) $L=3$, (b) $L=5$, (c) $L=9$, and (d) $L=17$

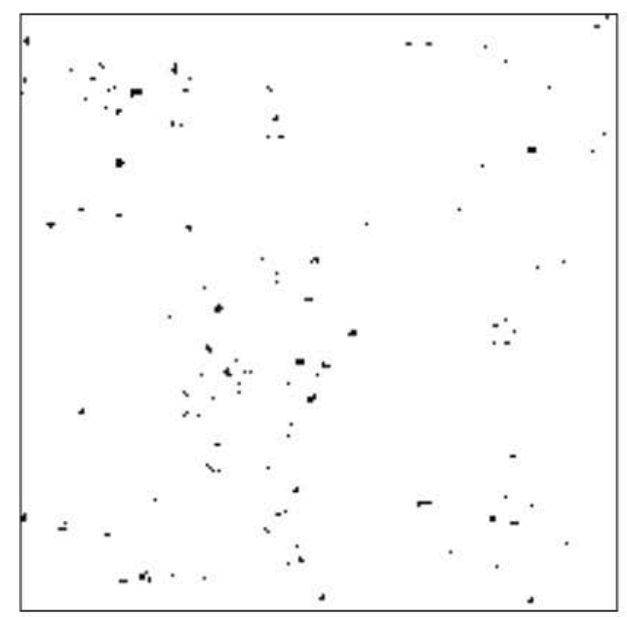

(a)

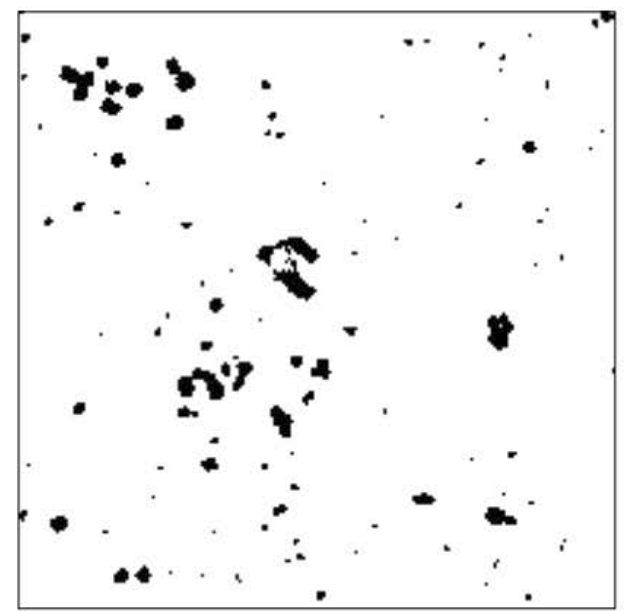

(c)

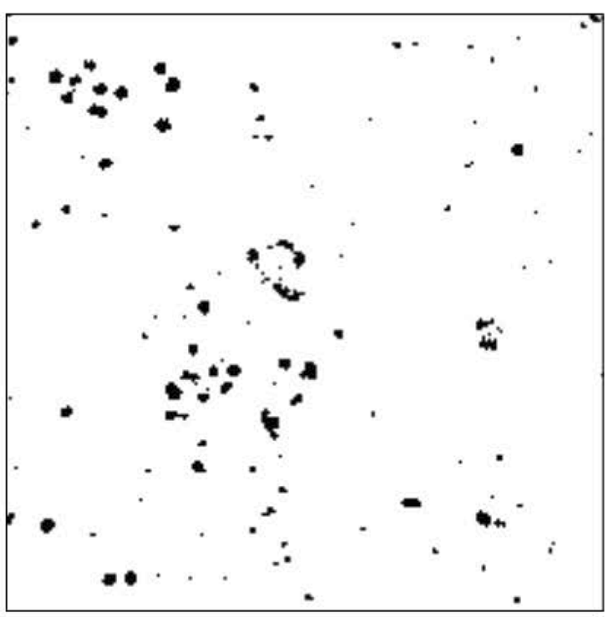

(b)

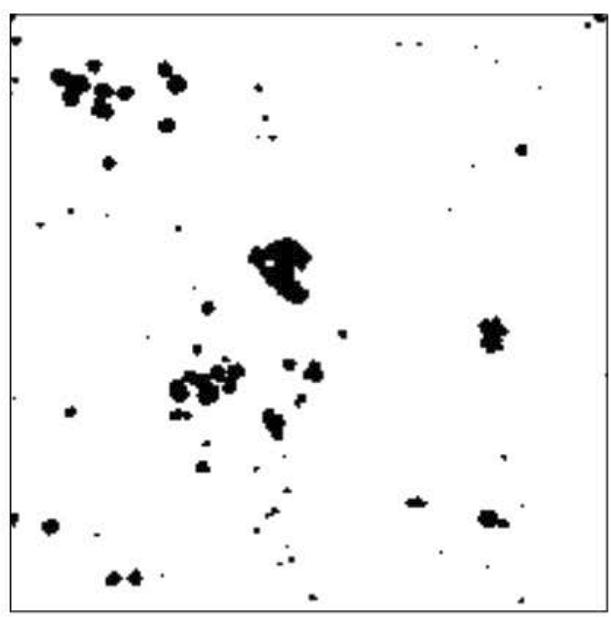

(d) (b) was processed and Fig. 10 shows the processing results with different $L$ values under $K=0.2$.

By observing these results, the noise is effectively filtered after the first step. But, on account of the inherent limitation of SprayCam, the particles occupy a very small area in the image, so the result obtained by fuzzy edge detection is more like a surface rather than an edge. Moreover, it can be found that when the $\mathrm{L}$ value is small, the algorithm is able to identify very small particles, as shown in Fig. 10(a) and (b). For the larger particles, the result of using a smaller $L$ is not satisfactory. When the values of $\mathrm{L}$ increase, the algorithm can identify larger particles but not the smaller ones. Therefore, fuzzy operations can eliminate the noise very well. However, information of the object itself will be lost if the degree of blur increases.

In order to keep the detection results both for smaller particles and larger particles, the results should be merged. Therefore, the third step will superimpose the detection

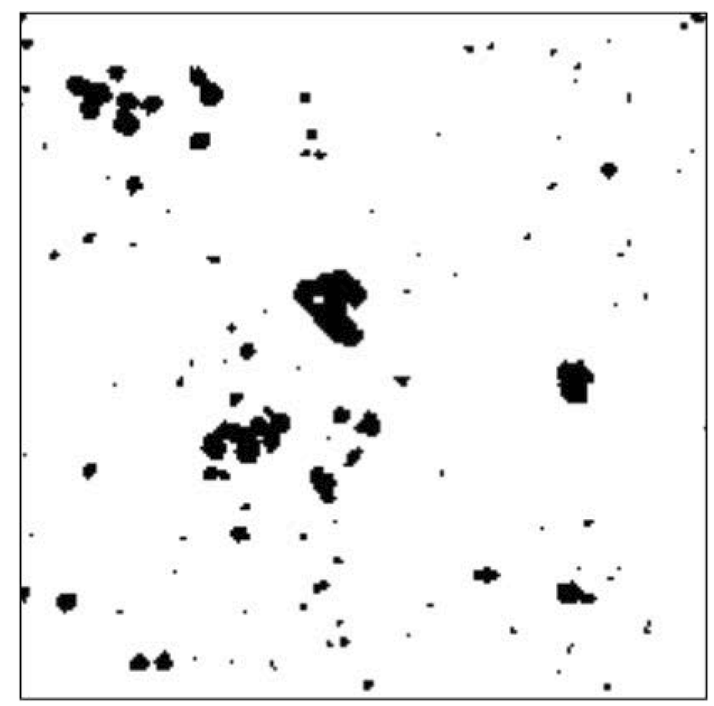

Fig. 11 Result obtained by superimposing the results of all fuzzy edge lengths when $K=0.2$ 
results obtained for different $\mathrm{L}$ values into a single result. If a pixel is regarded as an object resulting from any $L$ value, the changed pixel is considered an object and is marked black. The remaining pixels are white. The result obtained by this method is shown in Fig. 11. The detection result is similar to the result of the image segmentation algorithm.

\section{Parameters decision}

The algorithm's framework has changed from a singlelevel detection to a multi-level detection composed of different $L$ length. The number of levels is defined as $n$. $L$ can be arranged from small to large to establish a relationship between $\mathrm{n}$ and $L$ when $n=1, L$ gets its minimum value. When $n$ increases, $L$ increases accordingly. Thus, the level of the fuzzy operation increases. It can be seen in Fig. 10 the relationships between the increase of the degree of blur and the complete detection of larger particles. In other words, when the window of length $L$, the degree of blur is much greater than the diameter of the particle imaging. For larger diameter particles, the results obtained are very similar to the previous comparison. However, the information of adjacent small particles will be mixed together after the blur, which will lead to the false detection results. Therefore, the maximum value of $\mathrm{L}$ (defined as $L_{\text {max }}$ ) should be close to the maximum particle size of the particles. $D_{\max }$ is defined as the biggest granularity of the particles, and IR is the image resolution of SprayCam. Thus, $\left(\frac{D_{\max }}{I R}\right)$ is the maximum number of pixels due to particles. As shown in Fig. 8, the $L$ should be an odd number and the $L_{\text {min }}$ cannot be smaller than 3 . Eq5 is defined to ensure that $L_{\max }$ is an odd number close to the maximum number of pixels for the particles.

$L_{\max }=\frac{D_{\max }}{I R} / 2 * 2+1$

After obtaining $L_{\max }$, the highest level of fuzzy operations, the number of fuzzy operations $n_{\max }$ should be defined. In addition, considering the detection results of particles of different sizes, smaller particles are more likely to be ignored by the algorithm. Therefore, when $L$ is a small value, more tests need to be performed to avoid information loss. When $\mathrm{L}$ is large, since the information of medium-sized particles is unlikely to be ignored by the algorithm, the number of detections can be reduced accordingly. Thus, different levels of window sizes (in multiples of 2 as the unit) are selected as the detected correspondence between $\mathrm{L}$ and $\mathrm{n}$. Therefore, the maximum number of levels $n_{\max }$ is:

$n_{\text {max }}=\log _{2}\left(L_{\max }\right)$

For each fuzzy operation, the length L of the window follows the result of $\mathrm{Eq} 6$ :
$L=2^{n}+1$

Therefore, for each fuzzy level, the formula of Eq 3 and Eq 4 changes to:

$G_{n}(i, j)=\frac{1}{\mathrm{~L}^{2}} \times \sum_{i=x-\frac{L-1}{2}}^{x+\frac{L+1}{2}} \sum_{j=y-\frac{L-1}{2}}^{y+\frac{L+1}{2}} F(i, j)$

$g_{n}(i, j)=\frac{G_{n}(i, j)-F(i, j)}{G_{n}(i, j)}$

If the result $g_{n}(x, y)$ of the studied pixel in a fuzzy level is greater than the specific value $K$, then this pixel is considered as an object and marked as Black.

To explore the threshold value $K$, it is necessary to use a non-object image. That means the images need to be captured when no object is in the measure range. The traversal method is used to search the appropriate value of $K$ values which is between 0.05 and 0.3 . For non-object image detection, the gray value of all the pixels should be 255 in the segmentation result. Among these qualified $K$, the algorithm will choose the smallest value as the threshold. It is worth noting that when the SprayCam parameters are changed, the $K$ value will be changed accordingly.

Thus, the $K$ values in this paper are based on the SprayCam parameters shown in Table 1. The result of $K$ is 0.131 .

After defining $K=0.131$, the algorithm was applied to test the detection performance. Figure12 (a, c, e, g) shows the original image. The detection results are shown in Fig. 12(b, d, f, h), respectively. For the fixed particles (Fig. 12f, h), particles and dust were detected perfectly. For the plasma spray images (Fig. 12a, c), the results (Fig. 12b, d) show that the algorithm can detect the particles which are out of the plasma jet. Meanwhile, although the influence of the plasma jet can be circumvented in the preprocessing, it still suffers from many restrictions. When small powders enter the plasma jet, their imaging appears overexposed, resulting in detail lost. In that specific case, the algorithm cannot work in this situation perfectly. This problem should be a physical level limit, which cannot be solved by optimizing the image processing.

Regarding the result of the fixed particle image (Fig. 12f, h), the detection of the image near the focal plan (Fig. 12f) is quite good, and the particles and noise are well distinguished, thus proving the high accuracy of the developed algorithm. When the particles are far away from the focal plan (Fig. 12h), the algorithm no longer recognizes dust and smaller particles. However, by looking at the original image, the information representing these small objects has been masked by noise. This is not a limitation of the algorithm itself but a loss of information due to Out of Focus Photography. Meanwhile, the large diameter objects can also be detected by the algorithm without a 


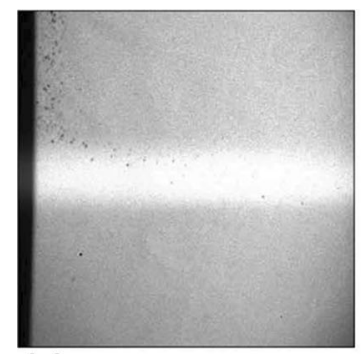

(a)

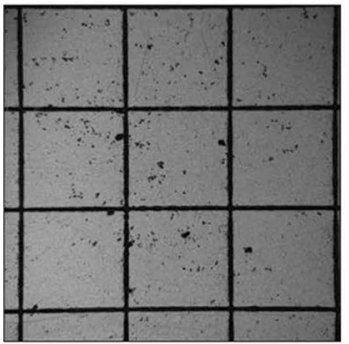

(e)

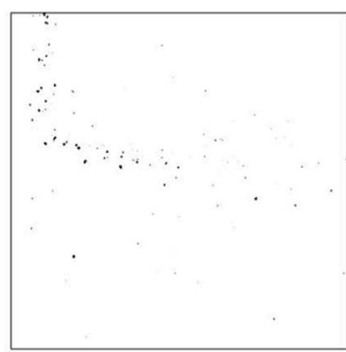

(b)

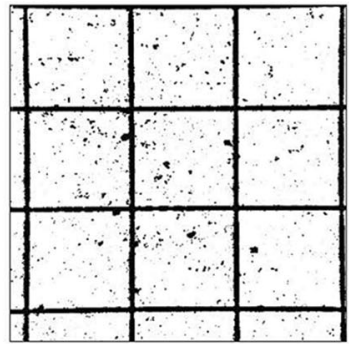

(f)

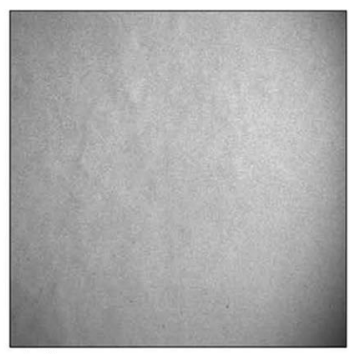

(c)

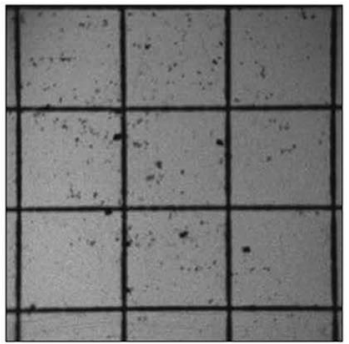

(g)

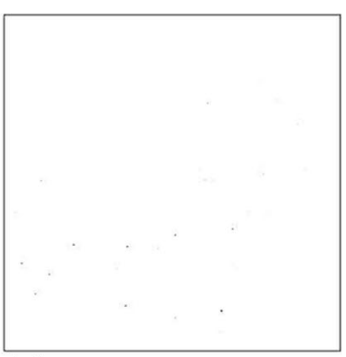

(d)

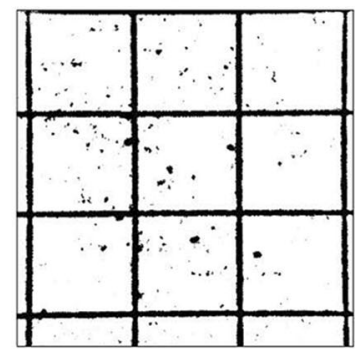

(h)

Fig. 12 Original image and corresponding detection result after particle detection when $K=0.131$

problem. This confirms that the algorithm still performs well in the case of objects far from the focal plan.

Generally, this algorithm uses preprocessing calculations to reduce most of the defects caused by environment and equipment limitations. It can also expand the range of valid information in the original image. The detection can obtain the high-sensitivity results for particles of different sizes, no matter where the particles are located. It will provide a good basis for future research.

\section{Discussion and Comparison with Other Methods}

There are many algorithms dedicated to objects detection. To demonstrate the advantages of the proposed algorithm, it is necessary to compare the results with other algorithms. Since the algorithm is good at preprocessing, the comparison will be done only on object detection. An image is created for this comparison, consisting of four preprocessed images, as shown in Fig. 13(a). Considering that the sprayed particles may be distant from the focal plan (close or far) in the same frame, the images obtained with the fixed particles of various distances are combined altogether. In this part, each specific algorithm will be performed using the edge detection algorithm, and the $\mathrm{K}$-means clustering will be used to compare the advantages and disadvantages of each algorithm.

Edge detection is currently the primary method used for object detection (Ref 23). Among the different methods, the performance of Canny edge detection is very good (Ref
24). This algorithm uses gradient modifications to determine the position of the edge. Gaussian blur step is defined as a preprocessing step, so that the Canny edge detection is resistant against noise ( $\operatorname{Ref} 25$ ). Thus, it was chosen for this comparison. Both Canny algorithm and the proposed multilevel fuzzy edge algorithm will be applied to detect the particle. Figure 13(c) presents the result of Canny detection.

By comparing Fig. 13(b) (obtained by proposed algorithm) and Fig. 13(c) (result of Canny algorithm), both results are acceptable if the particles are close to the focal plan. However, when the particles are far away, Canny edge detection fails to detect the particle edges, but the proposed algorithm still works for this situation.

Besides, another image segmentation algorithm called $\mathrm{k}$-means clustering is also a good challenger (Ref 26) to detect objects. Its result is similar to that of multi-level fuzzy detection algorithm. Supposing $k=2$ as variable of $k$ means clustering, one class concerns the background, and the other class is the object.

From the comparison between Fig. 13(b) and (d) (results issued from k-means clustering), the results of these two algorithms are similar. K-means clustering gives good result either for particles near the focal plane or large particles far from the focal plane. On the contrary, when the gray value of particles or dust is close to the background, k-means clustering cannot fully work. In this condition, the performance of proposed multi-level fuzzy detection algorithm is still the best. 
Fig. 13 (a) Original image, (b) fuzzy particle detection results in this paper, (c) Canny edge detection results, (d) $k$ means clustering results when $k=2$

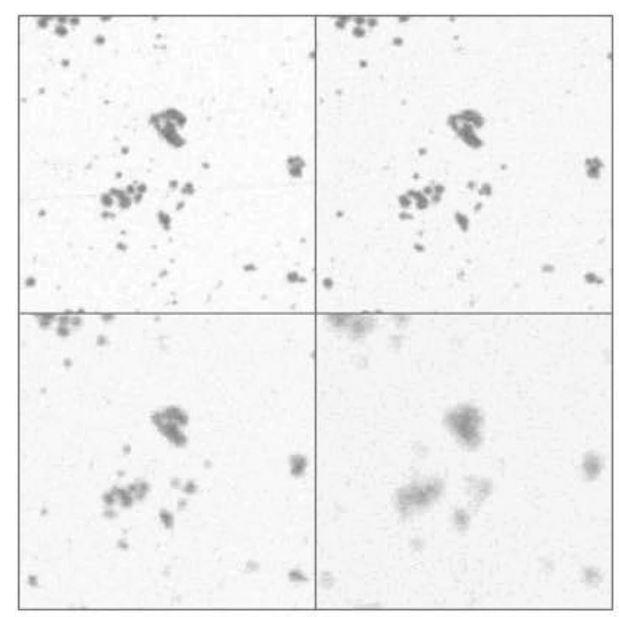

(a)

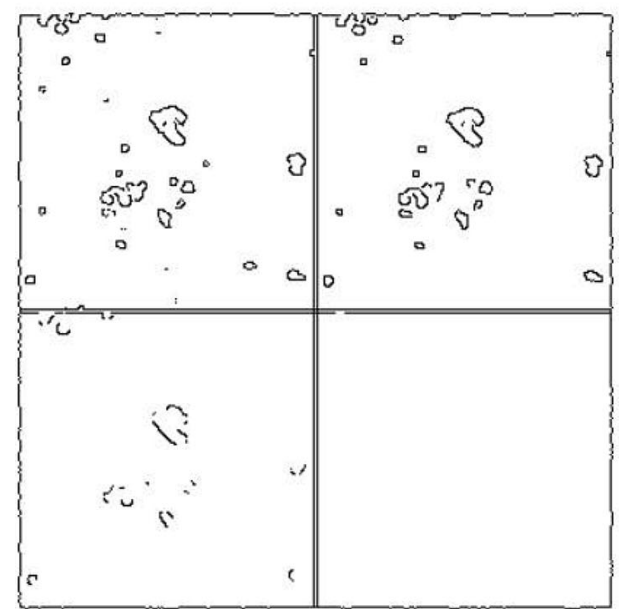

(c)

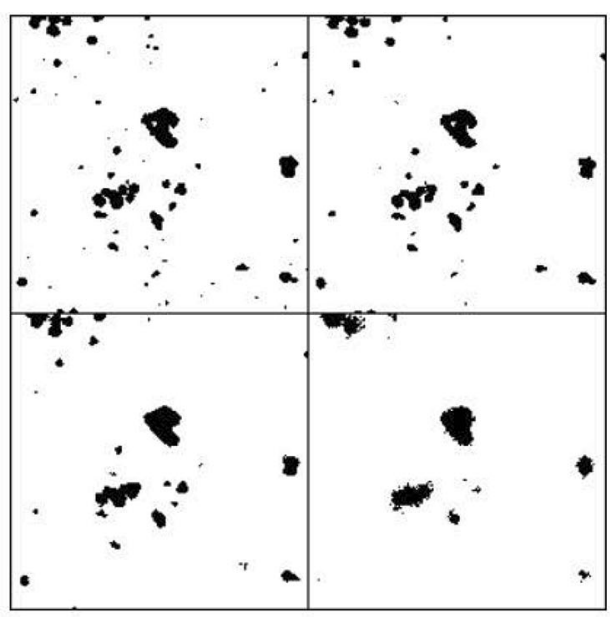

(b)

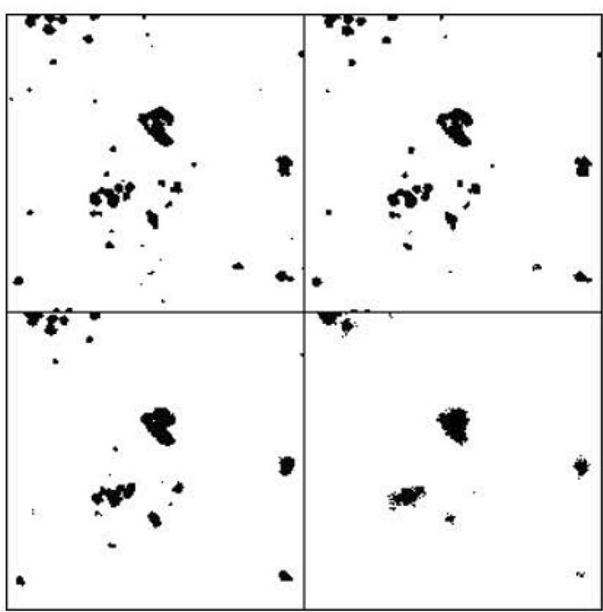

(d)

\section{Conclusions}

This paper introduced a complete method to identify inflight particles during thermal spraying. An alternative experiment (fixed particles images) has also been proposed to obtain data (Particle images) at different distances from the focal planes. The results show:

- A good elimination of the negative effects of the plasma jet.

- An effective reduction of the vignette on the images.

- A precise extraction of in-flight particles from noise.

This research provides the possibility to study in-flight particles based on short exposure and high-noise images, thereby making it possible to study particles far away from the focal plane.

This research uses multiple level fuzzy edge detection to get segmentation image results rather than the traditional edge detection methods. The comparison results confirm that this work avoids the risk of open edges in object detection and gives good results in any case. This research can be applied to any noninvasive imaging systems. With the help of image processing technology, the algorithm can identify the particles imaged in the observation device very well. The computer intervention can largely free up the human and provide a good basis for more in-depth studies such as particle velocimetry, particle size and position measurement with little reliance on specific devices. For example, in the case of plasma spraying as a potential in the paper, observing the nozzle position and the particles in the flame was almost impossible in the previous studies. In this work, particles can be observed successfully and accurately owing to help of algorithm. It is useful for other studies in the field. For example, it will give more validation data for the work of simulation and will give more learning data to the artificial intelligence and thus make the optimized spraying parameters more reliable.

In addition, the speed of technology iteration is often limited by the equipment. Previously, particle velocimetry relied on highly customized devices such as DPV 2000, 
which perform very good in their own fields. However, as new needs of research appear, they were not able to reach these goals quickly. Therefore, the idea of developing noncustomized devices through algorithms can be a good way to reduce the dependence on devices in the field and expand the possibilities. In other words, the intervention of algorithms can address the needs in research faster and thus accelerate the development of the measurement need in thermal spray.

Acknowledgments The author gratefully acknowledges Christian ADAM and Joël CHAUVELOT for their help in the plasma experiment.

\section{References}

1. A. Schutze, J.Y. Jeong, S.E. Babayan, J. Park, G.S. Selwyn and R.F. Hicks, The Atmospheric-Pressure Plasma Jet: A Review and Comparison to Other Plasma Sources, IEEE Trans. Plasma Sci., 1998, 26(6), p 1685-1694.

2. I. Brown, Vacuum-Arc Ion Sources, Rev. Sci. Instrum., 1994, 65(10), p 3061-3082.

3. P. Fauchais, Understanding Plasma Spraying, J. Phys. D-Appl. Phys., 2004, 37(9), p R86-R108.

4. K. Degroot, R. Geesink, C. Klein and P. Serekian, Plasma Sprayed Coatings of Hydroxylapatite, J. Biomed. Mater. Res., 1987, 21(12), p 1375-1381.

5. P. Fauchais and A. Vardelle, Thermal Plasmas, IEEE Trans. Plasma Sci., 1997, 25(6), p 1258-1280.

6. P. Fauchais and M. Vardelle, Sensors in Spray Processes, $J$. Therm. Spray Technol., 2010, 19(4), p 668-694.

7. N. Zhang, D. Lin, Y. Li, Y. Zhang, M. Planche, H. Liao, C. Coddet and F. Dong, In-Flight Particle Characterization and Coating Formation under Low Pressure Plasma Spray Condition, J. Iron Steel Res. Int., 2017, 24(3), p 306-312.

8. S. Dyshlovenko, L. Pawlowski, B. Pateyron, I. Smurov and J.H. Harding, Modelling of Plasma Particle Interactions and Coating Growth for Plasma Spraying of Hydroxyapatite, Surf. Coat. Technol., 2006, 200(12-13), p 3757-3769.

9. M. Friis, C. Persson and J. Wigren, Influence of Particle In-Flight Characteristics on the Microstructure of Atmospheric Plasma Sprayed Yttria Stabilized ZrO2, Surf. Coat. Technol, 2001, 141(2-3), p 115-127.

10. G. Mauer, R. Vaßen and D. Stöver, Comparison and Applications of DPV-2000 and Accuraspray-G3 Diagnostic Systems, J. Therm. Spray Technol., 2007, 16(3), p 414-424.

11. J. Vattulainen, J. Knuuttila, T. Lehtinen, T. Mantyla, and R. Hernberg, "In-Flight Particle Concentration and Velocity
Measurements in Thermal Spraying Using a Non-Intensified CCD Camera," Thermal Spray, Vols 1 and 2: Meeting the Challenges of the 21st Century, C. Coddet, Ed., (Materials Park), Asm International, 1998, p 767-772.

12. R. Boie and I. Cox, An Analysis of Camera Noise, IEEE Trans. Pattern Anal. Mach. Intell., 1992, 14(6), p 671-674.

13. J.C. Russ, Image Processing, Computer-Assisted Microscopy: The Measurement and Analysis of Images, J.C. Russ, Ed., (Boston, MA), Springer US, 1990, p 33-69, https://doi.org/10.1007/ 978-1-4613-0563-7_3.

14. F.-W. Bach, K. Möhwald, T. Rothardt, J. Prehm, L. Engl, K. Hartz and B. Drößler, Particle Image Velocimetry in Thermal Spraying, Mater. Sci. Eng. A, 2004, 383(1), p 146-152.

15. R.J. Adrian, Particle-Imaging Techniques for Experimental Fluid Mechanics, Annu. Rev. Fluid Mech., 1991, 23(1), p 261-304.

16. B. Ganapathisubramani and N.T. Clemens, Effect of Laser Pulse Duration on Particle Image Velocimetry, AIAA J. Am. Inst. Aeron. Astron., 2006, 44(6), p 1368-1371.

17. J. Vattulainen, E. Hämäläinen, R. Hernberg, P. Vuoristo and T. Mäntylä, Novel Method for In-Flight Particle Temperature and Velocity Measurements in Plasma Spraying Using a Single CCD Camera, J. Therm. Spray Technol., 2001, 10(1), p 94-104.

18. V. Srinivasan, M. Friis, A. Vaidya, T. Streibl and S. Sampath, Particle Injection in Direct Current Air Plasma Spray: Salient Observations and Optimization Strategies, Plasma Chem. Plasma Process., 2007, 27(5), p 609-623.

19. "Control Vision Inc," n.d., http://www.controlvisioninc.com/pro ducts/thermalsprayspraycam.html. Accessed 22 January 2021.

20. D.B. Goldman, Vignette and Exposure Calibration and Compensation, IEEE Trans. Pattern Anal. Mach. Intell. IEEE, 2010, 32(12), p 2276-2288.

21. S.Y. Lee and Y.D. Kim, Sizing of Spray Particles Using Image Processing Technique, KSME Int. J., 2004, 18(6), p 879-894.

22. J. Wu, Z. Yin and Y. Xiong, The Fast Multilevel Fuzzy Edge Detection of Blurry Images, IEEE Signal Process. Lett., 2007, 14(5), p 344-347.

23. J. Canny, A Computational Approach to Edge-Detection, IEEE Trans. Pattern Anal. Mach. Intell., 1986, 8(6), p 679-698.

24. D. Ziou and S. Tabbone, Edge Detection Techniques-an Overview, Pattern Recognition and Image Analysis C/C of Raspoznavaniye Obrazov I Analiz Izobrazhenii, 1998, 8, p 537-559.

25. X.-L. Zhao, F. Wang and M.K. Ng, A New Convex Optimization Model for Multiplicative Noise and Blur Removal, SIAM J. Imaging Sci., 2014, 7(1), p 456-475.

26. S. Ray and R.H. Turi, "Determination of Number of Clusters in K-Means Clustering and Application in Colour Image Segmentation," Calcutta, India, 1999, p 137-143.

Publisher's Note Springer Nature remains neutral with regard to jurisdictional claims in published maps and institutional affiliations. 